\title{
Policy Brief: Proposed Solutions to Implement the Priorities of the Sendai Framework to Reduce the Risk of Accidents: A Policy Brief
}

\author{
Mahmood Nekoiemoghadam ${ }^{1}$ (D), Seyed Mobin Moradi ${ }^{*}$ (D), Hamid Reza Khankeh (D) ${ }^{2}$, Gholam Reza Masoumi $^{3}$, Amir Nejati $^{4}$, Ali Mehrab $^{\circ}$ \\ Tavana $^{5}$, Mohammad Hossein Yarmohammadian ${ }^{6}$, Ahmad Jonidi Jafari ${ }^{3}$ \\ 1. Health in Disaster and Emergencies Research Center, Institute for Futures Studies in Health, Kerman University of Medical Sciences, Kerman, Iran. \\ 2. Health in Emergency and Disaster Research Center, University of Social Welfare and Rehabilitation Sciences, Tehran, Iran. \\ 3 Department of Environmental Health Engineering, School of Health, Iran University of Medical Sciences, Tehran, Iran. \\ 4. Department of Emergency Medicine, Prehospital and Hospital Emergency Research Center, School of Medicine, Tehran University of Medical Sci- \\ ences, Tehran, Iran. \\ 5. Health Management Research Center, Baqiyatallah University of Medical Sciences, Tehran, Iran. \\ 6. Health Management and Economics Research Center,School of Management and Medical Information Sciences, Isfahan University of Medical Sci- \\ ences, Isfahan, Iran
}



Cftat On: Nekoiemoghadam M, Moradi SM, Khankeh HR, Masoumi GhR, Nejati A, Mehrabi Tavana A, et al. Proposed Solutions to Implement the Priorities of the Sendai Framework to Reduce the Risk of Accidents: A Policy Brief. Health in Emergencies and Disasters Quarterly. 2020; 6(1):57-62. http://dx.doi.org/10.32598/hdq.6.1.336.1

doi': http://dx.doi.org/10.32598/hdq.6.1.336.1

\section{(c) (i) (5)}

Article info:

Received: 08 Oct 2019

Accepted: 14 Sep 2020

Available Online: 01 Oct 2020

\section{Keywords:}

Disasters, Sendai framework, Disaster risk reduction, Policy brief

\section{A B STRACT}

Accidents and disasters impose enormous costs on governments and nations each year, as well as causing great suffering to people affected by various disasters around the world. Today, accidents and disasters account for a large portion of government resources and programs. Iran is no exception to this rule. It is one of the ten most populated countries globally and the fourth most troubled country in Asia in terms of natural disasters. Therefore, understanding the risks as a basis for future planning is vital. Reducing disaster risk as a global priority requires the collective efforts of policymakers, managers, professionals, and all stakeholders in this field. The Sendai Framework is the most critical UN disaster risk reduction strategy (20152030). Considering the importance of the mentioned cases, a group of experts came together and discussed the solutions for implementing the Sendai Framework in Iran using the focus group discussion method. Finally, they prepared the present policy brief. One of the most important strategies is to study the history of risk in different parts of the world and Iran and prepare a risk zoning map, form a transnational and national organization. This map should delegate each authority to a particular organization for risk management, emphasize the correct understanding and upgrade the scientific level of people towards risk awareness. It must pay particular attention to create a culture in the field of risk awareness, analyzing stakeholders to attract support, and create a national information network. The map ought to develop a comprehensive risk management program, expanding education at the level of managers and society, creating and upgrading a quick alert system, and comprehensive preparedness plans. Finally, it can be said that the Sendai Framework is one of the most important documents published to plan for disasters. This planning is not just in the response phase but must be applied at all the crisis management cycle levels.

\section{* Corresponding Author:}




\section{Introduction}

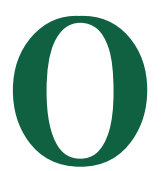

ne of the significant challenges throughout human history is dealing with natural disasters and protecting life and property [1]. Today, accidents and disasters account for many government resources and programs - the media casts massive information on various events worldwide. Climate change, human manipulation in nature, and the rapid growth of technology have increased the rate of accidents and people's vulnerability. Statistics show the growing number of accidents around the world. Every year, accidents and disasters inflict heavy casualties and financial losses on governments and nations [2]. By definition, accidents and disasters disrupt normal social activities, surpass the affected area's capacity to cope, and cause casualties and financial losses.

Effective management of these destructive events depends on predicting the consequences and problems of accidents and planning to respond effectively to the questions caused by them [3] — one of the issues that most cities of the world are involved in natural disasters. Natural disasters (especially earthquakes), which are often silent but potentially damaging, cause an average of more than 150000 casualties and more than $\$ 140$ billion financial losses annually in countries, especially in developing countries [4].

Iran is one of the tenth most troubled countries globally and the fourth most troubled country in Asia in terms of natural disasters [5]. Located on the earthquake belt, Iran, with a $1164819 \mathrm{~km}^{2}$ land area, constantly faces natural crises. The high prevalence of seismic faults in some country regions is notable; Zarand and Bam are earthquakes examples. Out of about 300 cities in the country, including the most populated areas, $77 \%$ of them are located on seismic faults, and $35 \%$ of Iranian towns are exposed to floods, storms, and coastal waves [2]. Statistics show that more than 109000 people have been killed and 150000 injured over the past 40 years and that Iran is not in an excellent position to deal with disasters [6]. During these years, 53 million people have been affected by disasters [7]. On December 26, 2003, the Bam earthquake killed more than 26000 and injured more than 30000. This shows that the construction in the fault locality and ignoring the construction code related to the type of risk in the region has led to massive loss of life [8].

Reducing disaster risk as a global priority requires the collective efforts of policymakers, managers, professionals, and all stakeholders in this field. In this regard, global knowledge and experience can be considered a practical step in improving the country's understanding of unexpected events.
The Sendai Framework is the most critical UN disaster risk reduction strategy (2015-2030) [9]. The Sendai Framework for Disaster Risk Reduction was adopted at the Third United Nations World Conference in Sendai, Japan, on March 18, 2015. It is a tool that replaced the Hugo Framework (2005-2015) (entitled Resilience of Nations and Communities to Disasters). The Sendai Framework is based on the elements that strengthen and ensure the continuity of work by governments and other stakeholders in line with the Hugo Framework (HFA). Many experts point to the most significant changes made in this framework: the strong emphasis on accident risk management instead of accident management, identifying 7 sub-global goals, reducing accident risk as an expected outcome, focusing on new risk prevention, reduce existing risks, strengthen resilience, as well as a set of guiding principles, including the primary responsibility of governments to prevent and reduce the risk of accidents, and the interaction of society as a whole and all government agencies. Besides, the territory of targeted accident risk reduction, with a focus on both natural and man-made disasters, as well as environmental, technological, and biological risks, has dramatically expanded and improved health resilience in all sectors [9].

\section{Strategies in the Sendai Framework}

A- Recognizing and understanding the risk of disasters (first priority):

Disaster risk management should be based on understanding disaster risk in all dimensions, including vulnerability, capacity, exposure of people, assets and characteristics of risks, and the environment.

B- Strengthen governance and leadership for disaster risk management (second priority);

Disaster risk management at all national and global levels is critical to disaster risk management in all sectors. Based on this, it is possible to ensure public policies and national and local laws and regulations to define roles and responsibilities, and then guide and encourage the public and private sectors.

C- Investing in disaster risk reduction to create resilience (third priority);

Public and private investments in disaster prevention must reduce the risk of disasters through structural and non-structural measures to improve the resilience of individuals, communities, countries, and their assets and the environment. These investments can be a source of 
initiative, growth, and job creation. Such actions are essential for saving lives, preventing and reducing damages, which is also useful and ensures effective response and recovery.

D- Improving disaster preparedness for effective response and rehabilitation (fourth priority) [10].

\section{Problem analysis}

The Sendai Framework is a wholly and comprehensively designed document with specific goals and strategies for all communities. However, its implementation is left to the communities to develop operational and corrective programs based on their particular conditions [10]. It can be said that the document is a beacon for governments and societies to show them the way, and they must implement it themselves. Iran, with its long history of disaster, is no exception.

In this regard, to prepare solutions for the implementation of the Sendai Framework, a working group consisting of members of the Board of Health in Disasters and a wide range of experts in the field of risk management, urban management, geology, meteorology, and health students in accidents and disasters gathered at Kerman University of Medical Sciences to discuss and exchange views on the agreed subjects and advisory opinions in 2016. The method of collecting information and ideas of experts in this working group was focus group discussion. Similarly, based on the logic of focus group discussion, one person as an interviewer and one person as a writer presented the issues and then documented them [11]. In this meeting, which was led and facilitated by Dr. Hamidreza Khankeh, each of the Sendai Framework strategies was discussed separately. In this method, the main topic was written on the board, and then the opinions of experts about the area in question were expressed. After all participants' agreement on the issues raised, each strategy's final solutions were recorded and written. Finally, the proposed solutions were prepared in the form of a list of each process and discussed below.

\section{Suggested solutions}

A- Proposed solutions of the first strategy (recognizing and understanding the risk of disasters).

1. Studying the history of danger in all parts of the world and Iran based on different country regions and preparing and compiling a risk zoning map.
2. Establishing and strengthening research centers in the field of disasters and their networking.

3. Using scientific and valid methods for risk, recognizing and explaining its concepts and various dimensions, and using scientific metrics to analyze, describe, measure, and prioritize risks.

4. Production and introduction of indigenous tools appropriate to the country's resources, as well as the development of risk recognition standards, to conduct annual assessments of risk recognition (such as converting Hospital Safety Index (HSI) to Farsi version (FHSI).

5. Forming specialized risk groups and using elites' opinions of different fields to create common languages and words at the macro level and designing and compiling risk maps.

6. Establish a national and supra-factional organization or delegate authority to an existing organization to focus on the necessary measures in risk recognition and attract the participation and support of high-ranking officials of the country and the private sector.

7. Planning, establishing, and developing integrated information infrastructures to identify risks, profile them, capacities, and vulnerabilities in various fields.

8. Moving towards sustainable development in risk recognition (considering political, security, and economic concerns) and considering risk management in organizational missions.

9. Emphasizing, understanding, and upgrading people's scientific level towards risk awareness through educational programs and its localization based on risk recognition and spatial planning studies.

10. Designing educational programs at all levels, from elementary school to university, to create a correct understanding of risk.

11. Paying particular attention to culture building in recognizing risks at all levels, from the country's highest levels to organizations and finally the people, using mass media and making related programs.

12. Establishing social models to increase risk awareness and promote preventive and pragmatic attitudes instead of destiny beliefs using experts and a communitybased approach to change attitudes. 
13. Developing non-governmental organizations (NGOs) to recognize the risk and create public sensitivity by acknowledging and understanding the risk through focusing on society's needs.

14. Applying 5 principles of risk perception with components:

a. Prepare a risk zoning map.

b. Use the maps for all development and risk management programs.

c. Document disasters and disseminate results to individuals in the community.

d. Involve people in disaster-related matters.

e. Educate public.

B- Proposed solutions of the second strategy (strengthening governance and leadership for disaster risk management).

1. Merger of similar organizations with the same responsibilities and create a single organization at the national level to integrate activities and develop an appropriate structure for risk management in the country and also to prevent the multiplicity of power centers.

2. Compilation and development of laws and regulations in the field of risk management in the spatial planning of the country, as well as the result of guaranteed solutions for the implementation of laws and the provision of solutions to facilitate the implementation of laws.

3. Entering the priorities of related international documents by the legislature into 5-year plans and strengthening the position of risk management in upstream documents.

4. Stakeholder analysis to seek support as well as provide significant and sustainable financial resources and strengthen the participatory approach of stakeholder organizations.

5. Creating a national information network to transfer information to relevant organizations.

6. Preparing and compilation of the conditions for holding the position of risk management and applying the principle of meritocracy in this position, as well as the need for management stability in this area.
7. Conducting comparative studies to extract a suitable model for Iran and also standardize guidelines and protocols.

8. Developing a comprehensive risk management plan and regular and continuous monitoring at the national level and emphasize being program-oriented rather than individualistic.

9. Use of specialized managers in the field of risk management and upgrade their scientific level as leaders of organizations.

10. Increasing the share of risk reduction budgets in the country's macro budgets.

11. Attention and emphasis on comprehensive risk management, including risk, capacity, and vulnerability.

C- Proposed solutions of the third strategy (investing in disaster risk reduction to create resilience).

1. Establishing correct and transparent relations with the people to explain the problems caused by disasters and encourage them to invest in this sector using facilitating legal levers (such as tax cuts, standard exemptions, development of incentive programs, etc.).

2. Expanding training at the managerial and community level and provide expert staff to encourage investment.

3. Making efforts to increase risk awareness and thus change managers' attitudes to encourage investment.

4. Considering appendix to reduce the impact of accidents on current and development programs and allocate a share of National Development Fund resources to minimize risks.

5. Using international experience to increase investment and raise public access to transparent and technical information, as well as post-accident comparative studies to compare the impact of investment.

6. Establishing Disaster Risk Reduction Support Fund and allocation of subsidies to disaster risk reduction activities.

7. Developing knowledge-based companies to carry out scientific activities to reduce the risk of disasters and also economic justification for investing in this field. 
8. Adopting laws for the investment of economic enterprises and polluting industries, as well as laws related to insurance and protection laws, as well as revision of construction laws and regulations.

9. Paying particular attention to industry and market to prevent the advertising of unsafe goods and services and encourage people and society to buy safe goods and safety equipment, and personal protection.

10. Adopting the law in the Islamic Consultative Assembly to force the government to invest in risk reduction measures and facilitate other sectors' participation, including non-governmental organizations (NGOs), private and charitable organizations, and international organizations in this field.

D- Proposed Strategies for the Fourth strategy (Improving Disaster Preparedness for Effective Response and Rehabilitation and Reconstruction).

1. Establishing and upgrading a rapid alert system and developing community-based early warning protocols.

2. Institutionalizing and strengthening the incident command system to support and coordinate work between the organizations in charge of crisis response.

3. Compilation of a comprehensive disaster preparedness plan and practice the program in regular intervals.

4. Developing disaster preparedness and response training programs for different groups from elementary levels to households and universities and preparation of training packages suitable for each group.

5. Paying particular attention to social networks and media's role to provide proper education to the people and transfer timely and correct information at the time of disasters.

6. Providing a suitable platform for strengthening and enriching information systems for technologies such as simulation, GIS, etc., to prepare risk maps, forecast, supply, and store the required resources.

7. Paying particular attention to the role of non-governmental groups such as Non-Governmental Organizations (NGOs), the private sector, and international organizations to measures to enhance preparedness.

\section{Conclusion}

The Sendai Framework is one of the most important documents published to plan for disasters. This planning is not just in the response phase but must be applied at all risk management phases. This document emphasizes four strategies, each of which can be the basis of societies' problems in dealing with disasters. At first glance, these strategies are very general. Still, using experts' opinions, an attempt has been made to consider the practical details of each strategy as much as possible.

Considering that Iran is one of the most troubled countries in the region and the world, and several organizations in the country are in charge of planning and dealing with accidents and disasters, each of the organizations can achieve the primary goals of the Sendai Framework by 2030 by applying the details of each strategy at the level of their organization and in cooperation with other organizations. This research requires integrated national management and information system, and collaboration and coordination is the key to achieve an integrated approach.

\section{Ethical Considerations}

\section{Compliance with ethical guidelines}

There were no ethical considerations to be considered in this research.

\section{Funding}

This research did not receive any grant from funding agencies in the public, commercial, or non-profit sectors.

\section{Authors' contributions}

Conceptualization and supervision: Mahmood Nekoiemoghadam and Hamid Reza Khankeh; Methodology: Hamid Reza Khankeh, Gholam Reza Masoumi and Seyed Mobin Moradi; Investigation, writing - original draft, and writing - review \& editing: All authors; Data collection: Gholam Reza Masoumi and Seyed Mobin Moradi; Data analysis: Seyed Mobin Moradi, Mahmood Nekoiemoghadam and Hamid Reza Khankeh.

\section{Conflict of interest}

The authors declared no conflict of interest. 


\section{Acknowledgments}

We would like to thank Kerman University of Medical Sciences and also specialized board members of Health in Emergency and Disasters

\section{References}

[1] Moghadam MN, Moradi SM, Amiresmaili M. Examining non-structural retrofitting status of teaching hospitals in Kerman against disasters. Electronic Physician. 2017; 9(5):4434-9. [DOI: 10.19082/4434] [PMID] [PMCID]

[2] Khankeh H. [Hospital preparedness in emergency and disasters: A national program (Persian)]. Tehran: Tehran University of Social Welfare and Rehabilitation Sciences Publications; 2013. http://darman.tums.ac.ir/Content/media/filepool3/2015/2/821.pdf

[3] Jahangiri K, Izadkhah YO, Lari A. Hospital Safety Index (HSI) analysis in confronting disasters: A case study from Iran. International Journal of Health System and Disaster Management. 2014; 2(1):44-9. https://www.ijhsdm.org/article. asp?issn $=2347-9019 ;$ year $=2014 ;$ volume $=2 ;$ issue $=1$; page $=44$; epage $=49$ ;aulast=Jahangiri

[4] Pourahmad A, Lotfi S, Faraji A, Azimi A. Investigating the dimensions of earthquake crisis prevention (case study of Babol). Urban and Regional Studies and Research. 2009; 1(1):1-24. https://www.sid.ir/en/journal/ViewPaper.aspx?ID=169718

[5] Ardalan A. I. R. Iran National Health Disaster and Emergency Response Operations Plan (EOP). Tehran: Akbarbarzin Publishing House; 2015. http://darman.tums.ac.ir/Content/media/ filepool3/2016/1/1509.pdf

[6] Ghafouri RR, Hosseini SM, Pouraghaei M. Are our hospitals safe against disasters? An evaluation of hospital safety index in Tabriz, Iran. Journal of Research in Clinical Medicine. 2018; 6(3):139-44. [DOI:10.15171/jarcm.2018.022]

[7] Ardalan A, Rajaei MH, Masoumi G, Azin A, Zonoobi V, Sarvar M. 2012-2025 roadmap of IR Iran's disaster health management. PLoS Currents. 2012; 4:e4f93005fbcb34. [DOI:10.1371/4f93005fbcb34] [PMID] [PMCID]

[8] Tatar M, Hatzfeld D, Moradi A, Paul A. The 2003 December 26 Bam earthquake (Iran), Mw 6.6, aftershock sequence. Geophysical Journal International. 2005; 163(1):90-105. [DOI:10.1111/j.1365-246X.2005.02639.x]

[9] United Nations Office for Disaster Risk Reduction. Sendai framework for disaster risk reduction 2015-2030 [Internet]. 2015 [Update 2015 March 18]. Available from: https://www.undrr.org/publication/sendai-framework-disaster-risk-reduction-2015-2030

[10] Kelman I, Glantz MH. Analyzing the Sendai framework for disaster risk reduction. International Journal of Disaster Risk Science. 2015; 6(2):105-6. https://search.proquest.com/docview/1691397441/fulltextPDF/E2CA16CC84144CE6PQ/1?accountid=34377

[11] Khosravi Sh, Abed Saeedi Zh. [Focus group, a data gathering method (Persian)]. Iran Journal of Nursing (IJN). 2011; 23(68):19-30. http://ijn.iums.ac.ir/article-1-910-en.html 